\title{
Novel method of intraoperative liver tumour localisation with indocyanine green and near-infrared imaging
}

\author{
Hui Jun Lim$^{1}$, BMed, MD, Adrian Kah Heng Chiow ${ }^{1}$, MBBS, FRCSE, Lip Seng Lee ${ }^{1}$, MB BCh BAO, FRCSE, \\ Siong San $\underline{\operatorname{Tan}}^{1}$, MBBS, FRCSE, Brian KP $\underline{G o h}^{2,3}$, MBBS, FRCSE, Ye Xin $\underline{K o h}^{2,3}$, MBBS, FRCSE, Chung Yip $\underline{C h a n}^{2,3}$, MBBS, FRCSE,
} Ser Yee $\underline{L e}^{2,3}$, MBBS, FRCSE

\begin{abstract}
INTRODUCTION Fluorescence imaging (FI) with indocyanine green (ICG) is increasingly implemented as an intraoperative navigation tool in hepatobiliary surgery to identify hepatic tumours. This is useful in minimally invasive hepatectomy, where gross inspection and palpation are limited. This study aimed to evaluate the feasibility, safety and optimal timing of using ICG for tumour localisation in patients undergoing hepatic resection.

METHODS From 2015 to 2018, a prospective multicentre study was conducted to evaluate feasibility and safety of ICG in tumour localisation following preoperative administration of ICG either on Day 0-3 or Day 4-7.

RESULTS Among 32 patients, a total of 46 lesions were resected: 23 were hepatocellular carcinomas (HCCs), 12 were colorectal liver metastases (CRLM) and 11 were benign lesions. ICG FI identified 38 (82.6\%) lesions prior to resection. The majority of HCCs were homogeneous fluorescing lesions (56.6\%), while CLRM were homogeneous (41.7\%) or rimenhancing $(33.3 \%)$. The majority $(75.0 \%)$ of the lesions not detected by ICG FI were in cirrhotic livers. Most ( $84.1 \%)$ of ICG-positive lesions detected were $<1 \mathrm{~cm}$ deep, and half of the lesions $\geq 1 \mathrm{~cm}$ in depth were not detected. In cirrhotic patients with malignant lesions, those given ICG on preoperative Day 0-3 and Day 4-7 had detection rates of 66.7\% and $91.7 \%$, respectively. There were no adverse events.

CONCLUSION ICG FI is a safe and feasible method to assist tumour localisation in liver surgery. Different tumours appear to display characteristic fluorescent patterns. There may be no disadvantage of administering ICG closer to the operative date if it is more convenient, except in patients with liver cirrhosis.
\end{abstract}

Keywords: hepatobiliary surgery, indocyanine green, near-infrared imaging, tumour localisation

\section{INTRODUCTION}

Surgical resection remains the primary potentially curative intervention for hepatocellular carcinoma (HCC) and certain secondary malignancies of the liver such as colorectal liver metastases (CLRM). During liver resection, palpation and intraoperative ultrasonography (IOUS) are essential tools to determine tumour location, guide transection planes and identify small tumours that are undetected on preoperative imaging. However, regardless of the surgical approach, detection of small and/or deep lesions and differentiating these from cirrhotic or dysplastic nodules in the liver using IOUS remain difficult.

In the past decade, minimally invasive liver resection has gained wider acceptance and demonstrated a beneficial role in the management of liver malignancies. ${ }^{(1,2)}$ One of the limitations of laparoscopic surgery remains the lack of feedback and inability to palpate the liver and tumour directly. This poses additional technical challenges during surgery, especially if the tumour is small or not immediately visible at the surface of the liver.

Indocyanine green (ICG) is a negatively charged, amphiphilic, water-soluble but relatively hydrophobic tricarbocyanine dye taken up exclusively by hepatocytes and excreted into bile without enterohepatic recirculation. ${ }^{(2)}$ In the 1970s, proteinbound ICG was found to emit fluorescence peaking at $840 \mathrm{~nm}$ under illumination with near-infrared light. ${ }^{(3)}$ The fluorescence intensity of protein-bound ICG was found to correlate with its concentration to approximately $0.25 \mathrm{mg} / \mathrm{mL}$, decreasing at higher concentrations due to absorption of near-infrared light by ICG. ${ }^{(4)}$ Fluorescence imaging with ICG has increasingly been applied as an intraoperative navigation tool and is gaining greater attention in tumour localisation in hepatobiliary surgery. ${ }^{(5,6)}$ As bile contains proteins such as albumin and lipoproteins that bind ICG, clear fluorescent images of the biliary tract can be obtained following intrabiliary injection of ICG. ${ }^{(7)}$ The fluorescence of ICG and its biliary excretion can be used for intraoperative identification of hepatic lesions and tumours, which can be useful for tumour localisation and anatomical liver resection. ${ }^{(8,9)}$ In 2008, fluorescence imaging following portal injection of ICG was first used as an intraoperative technique to identify hepatic segments and subsegments for anatomical hepatic resection. ${ }^{(10)}$ Subsequently, refinements in imaging techniques have enabled the use of ICG fluorescence imaging for visualisation of hepatic tumours, enabling more accurate and precise anatomic resection of the liver in hepatobiliary surgery. ${ }^{(11-14)}$ Furthermore, various pathological lesions show characteristic fluorescent patterns that aid in identification of tumours. Hence, ICG fluorescence imaging serves as a useful navigational tool for tumour localisation in hepatobiliary surgery. Detection of tumours with ICG fluorescence with the near-infrared (NIR) camera system

${ }^{1}$ Hepatopancreatobiliary Unit, Department of Surgery, Changi General Hospital, ${ }^{2}$ Department of Hepatopancreatobiliary and Transplant Surgery, Singapore General Hospital, ${ }^{3}$ Duke-NUS Medical School, Singapore

Correspondence: A/Prof Lee Ser Yee, Senior Consultant, Department of Hepatopancreatobiliary and Transplant Surgery, Singapore General Hospital, Outram Road, Singapore 169608. drlee@surgicalassociates.sg 
Table I. Factors determining detection of liver tumour with ICG-NIR imaging in vivo.

\begin{tabular}{|c|c|c|}
\hline Factors & Detectability & Remarks \\
\hline \multicolumn{3}{|l|}{ Pathology } \\
\hline Malignant ${ }^{(8,13)}$ & +++ & \multirow{3}{*}{$\begin{array}{l}\text { ICG positivity in a tumour is not a biological marker of malignancy but a surrogate } \\
\text { of abnormal bile clearance dynamics. Nonetheless, based on the literature, most } \\
\text { malignant tumours tend to retain ICG more and for a longer period compared to } \\
\text { benign lesions or abnormal liver tissue. Benign tumours can be ICG positive as } \\
\text { well if bile excretion dynamics are abnormal within or surrounding the tumour. }\end{array}$} \\
\hline $\begin{array}{l}\text { Dysplastic/intermediate/ } \\
\text { well-differentiated } \mathrm{HCC}^{(8,13)}\end{array}$ & ++ & \\
\hline Benign $^{(15)}$ & $+/-$ & \\
\hline \multicolumn{3}{|l|}{ Tumour size } \\
\hline Large $^{(16)}$ & $++/+++$ & \multirow{2}{*}{$\begin{array}{l}\text { Generally, the larger the tumour, the more ICG it retains, allowing it to be more } \\
\text { detectable on the liver surface compared to a smaller and/or deeper tumour. }\end{array}$} \\
\hline Small(17) & $+/-$ & \\
\hline \multicolumn{3}{|l|}{ Tumour depth } \\
\hline Subscapular/< $1 \mathrm{~mm}$ deep ${ }^{(11,18)}$ & +++ & \multirow{3}{*}{$\begin{array}{l}\text { The tissue penetration of the fluorescence emitted by ICG ranges from only } \\
5 \mathrm{~mm} \text { to } 10 \mathrm{~mm} \text {. If the tumour is too deep, even if the lesion is ICG positive, the } \\
\text { florescence will not be able to penetrate through the thick liver parenchyma } \\
\text { for in vivo NIR camera detection intraoperatively. Detectability depends on the } \\
\text { interplay between tumour size and depth from the liver surface. }\end{array}$} \\
\hline Superficial < $1 \mathrm{~cm}^{(19)}$ & ++ & \\
\hline Deep $\geq 1 \mathrm{~cm}^{(9,19)}$ & $+/-$ & \\
\hline \multicolumn{3}{|l|}{ Liver parenchyma } \\
\hline Normal ${ }^{(20,21)}$ & ++ & \multirow[b]{2}{*}{$\begin{array}{l}\text { The liver parenchyma and the lesion must have a significant contrast in } \\
\text { fluorescence levels if the tumour is to be detectable (if it is ICG positive). Similarly, } \\
\text { this depends on two factors and their interplay: the timing of administration } \\
\text { and the state of the liver parenchyma (fibrosis/cirrhosis/steatosis). In other } \\
\text { words, if the liver is cirrhotic and/or the ICG is administered too near to surgery, } \\
\text { ICG retention in the non-tumour liver parenchyma will be high or still high, } \\
\text { i.e. bright (as the liver is unhealthy and/or has not had enough time for the ICG } \\
\text { to clear). In this situation, even if the tumour is bright, it will not be detectable, as } \\
\text { the background is just as bright, thereby concealing the tumour. }\end{array}$} \\
\hline Cirrhotic $^{(17)}$ & $+/-$ & \\
\hline \multicolumn{3}{|l|}{ Time of ICG administration } \\
\hline$>4$ days from surgery ${ }^{(22)}$ & $++/+++$ & \\
\hline$\leq 3$ days from surgery ${ }^{(23)}$ & $+/++$ & \\
\hline
\end{tabular}

ICG: indocyanine green; NIR: near-infrared

is dependent on the interplay of several factors such as tumour pathology, tumour depth, tumour size and the state of the nontumorous liver parenchyma. These factors are summarised in Table I. ${ }^{(15-23)}$

As the local experience of intraoperative ICG use is limited, this study aimed to evaluate the feasibility and safety of using ICG for tumour localisation in patients undergoing hepatic resection. In addition, we evaluated the protocol of ICG administration, including the optimal timing of ICG administration.

\section{METHODS}

From August 2015 to April 2018, patients who underwent open or minimally invasive hepatectomy using ICG fluorescence imaging at Singapore General Hospital and Changi General Hospital, Singapore, were included in the study. The eligibility criteria included all patients undergoing liver resection (both minimally invasive and open approaches) for hepatic tumours and/or had ICG administered for preoperative assessment of liver function within seven days of surgery. ICG retention at 15 minutes $\left(\mathrm{ICG}_{\mathrm{R} 15}\right)$ is an indication of a liver's health: the higher the $\mathrm{ICG}_{\mathrm{R} 15}$, the less healthy the liver is. Patients for whom surgeons deemed ICG to be potentially useful for tumour localisation or identification were recruited. Exclusion criteria were having any known or previous reaction to ICG or Stage 3-5 renal impairment based on the NKF KDOQI (National Kidney Foundation Kidney Disease Outcomes Quality Initiative) stages of kidney disease. Patients underwent ICG administration on either preoperative day (PRD) 0-3 or PRD 4-7.

Once a suitable case was identified, the patient was counselled by the recruiting surgeon and/or principal investigator as well as the study coordinators. The case was logged in the study database and logbook after informed consent was obtained. An information booklet on ICG and the ICG tumour localisation procedure was provided to the patient for further reading. Patients received ICG on either PRD 0-3 or 4-7. Appointments were arranged to coincide with the pre-anaesthesia evaluation clinic or pre-admission testing dates to minimise inconvenience to patients and their caregivers.

Surgery by the primary surgeon proceeded as planned. Our liver resection techniques have been previously described. ${ }^{(24-26)}$ Fusion ICG fluorescence images were obtained using the NIR camera system, with the Karl Storz IMAGE SPIES1 ${ }^{\text {TM }}$ (Karl Storz, Tuttlingen, Germany) and HyperEye (Mizuho Medical, Tokyo, Japan) systems being used for laparoscopic and open cases, respectively. All participating surgeons underwent a tutorial prior to their first case. A Karl Storz technical support staff was on-site at the first case for any troubleshooting required. The NIR camera system was made available throughout the whole duration of 
the case. For minimally invasive cases, the surgeon could opt for another camera system according to his preference during other aspects of the surgery, such as parenchymal transection. The NIR ICG detection system was used for the ICG tumour detection aspect, and IOUS was utilised to confirm the lesion, evaluate its depth from the liver surface and mark the resection margins. The whole surgery was recorded for auditing and documentation purposes.

Upon completion of resection, the whole and bivalved specimens were also documented for their fluorescence pattern, tumour depth and adequacy of margins. All pictures were taken during normal light coupled with a corresponding image during NIR mode. These were printed and stored as both soft and hard copies. Video and images were stored in the study's dedicated secured portable hard drive and filed securely. For the purposes of this study, ICG positivity was defined as the tumour being positively detected by the NIR system in vivo intraoperatively, while ICG negativity was defined as the inability of the NIR system to detect the tumour. This could be due to a variety of reasons, such as the tumour not retaining ICG, the overlying or surrounding liver parenchyma (i.e. background) being too bright and thus concealing the tumour, or the tumour being too deep so that ICG fluorescence could not penetrate the overlying liver.

Patients' clinical information, including history, physical assessment, pre- and postoperative laboratory and radiology investigations, surgical data, and histopathological data, were prospectively collected from the respective hospitals' clinical electronic health record system (Citrix Version 5.1.1, Pharsight Corporation, Mountain View, CA, USA) and Operating Theatre Management (OTM) system (OTM Version 11.0, NovaHealth, Singapore). All information was maintained in a secure database server with password-protected access by authorised personnel and members of the study, according to institutional protected health information regulations. This study was conducted with the approval of our institutional review board committee.

For patient characteristics, continuous variables were summarised using median and range. Categorical variables were summarised by number and percentage of patients in each category. Categorical data and continuous data were compared using Fisher's exact test and unpaired $t$-test, respectively. Statistical analyses were performed using GraphPad Prism version 7.04 (GraphPad Software Inc, La Jolla, CA, USA). A p-value of $<0.05$ was considered to indicate statistical significance.

\section{RESULTS}

A total of 46 hepatic lesions were resected from 32 patients. Table II summarises the patients' characteristics, including distributions of the hepatic tumours, ICG regimen administration and pathological diagnosis of the lesions. Concomitant colorectal resection was performed in two patients. 11 benign tumours resected in this study were considered malignant or indeterminate following discussion of the preoperative images, including computed tomography and magnetic resonance images, at a multidisciplinary meeting. The median operative time and length of postoperative hospital stay were 246 (79-540) minutes and 4 (1-60) days, respectively. The patients recovered satisfactorily
Table II. Patient characteristics.

\begin{tabular}{|c|c|}
\hline Characteristic & Median (range)/no. \\
\hline \multicolumn{2}{|l|}{ Day of ICG administration } \\
\hline PRD 0-3 & 11 \\
\hline PRD 4-7 & 21 \\
\hline \multicolumn{2}{|l|}{ Surgical approach } \\
\hline Laparoscopic & 26 \\
\hline Open & 6 \\
\hline \multicolumn{2}{|l|}{ Depth of hepatic lesion from capsule ( $\mathrm{mm})$} \\
\hline \multicolumn{2}{|l|}{ Median } \\
\hline ICG positive & $0(0-6.0)$ \\
\hline ICG negative & $1.5(0-10.0)$ \\
\hline \multicolumn{2}{|l|}{ Mean } \\
\hline ICG positive & 1.6 \\
\hline ICG negative & 2.6 \\
\hline \multicolumn{2}{|l|}{ Pathological diagnosis } \\
\hline Hepatocellular carcinoma & 23 \\
\hline Grade 1 & 1 \\
\hline Grade 2 & 19 \\
\hline Grade 3 & 3 \\
\hline Colorectal liver metastasis & 12 \\
\hline Benign hepatic lesion & 11 \\
\hline Macroregenerative nodule & 3 \\
\hline Benign bile duct adenoma/hamartoma & 2 \\
\hline Nodular steatosis & 2 \\
\hline Angiomyolipoma & 1 \\
\hline Epithelioid haemangioendothelioma & 1 \\
\hline Infarct with perivenular necrosis & 1 \\
\hline Sclerosing haemangioma & 1 \\
\hline \multicolumn{2}{|l|}{ Pathological findings (liver parenchyma) } \\
\hline Cirrhosis & 15 \\
\hline Steatosis & 14 \\
\hline \multicolumn{2}{|l|}{ Postoperative outcome } \\
\hline Operative time (min) & $246(79.0-540.0)$ \\
\hline Postoperative stay (day) & $4(1-60)$ \\
\hline ICG-related adverse events (\%) & 0 \\
\hline 90-day mortality (\%) & 0 \\
\hline
\end{tabular}

ICG: indocyanine green; PRD: preoperative day

from surgery and had uneventful postoperative courses. No patients encountered ICG-related adverse events during administration and peri- or postoperatively.

The pathological characteristics of the 46 resected hepatic lesions are summarised in Table II. ICG fluorescence imaging identified $38(82.6 \%)$ of the lesions prior to resection, while the remaining $8(17.4 \%)$ were not detected. Among the 38 detected lesions, 22 tumours displayed a homogeneous pattern, ten tumours showed a heterogeneous pattern, and the remaining nine tumours showed rim enhancement (Fig. 1).

The various characteristics of the ICG-positive and ICGnegative groups are compared in Table III. The majority (56.5\%) of the HCCs were detected as homogeneous fluorescing lesions (Fig. 2), while CRLM were mainly (58.3\%) detected as either 

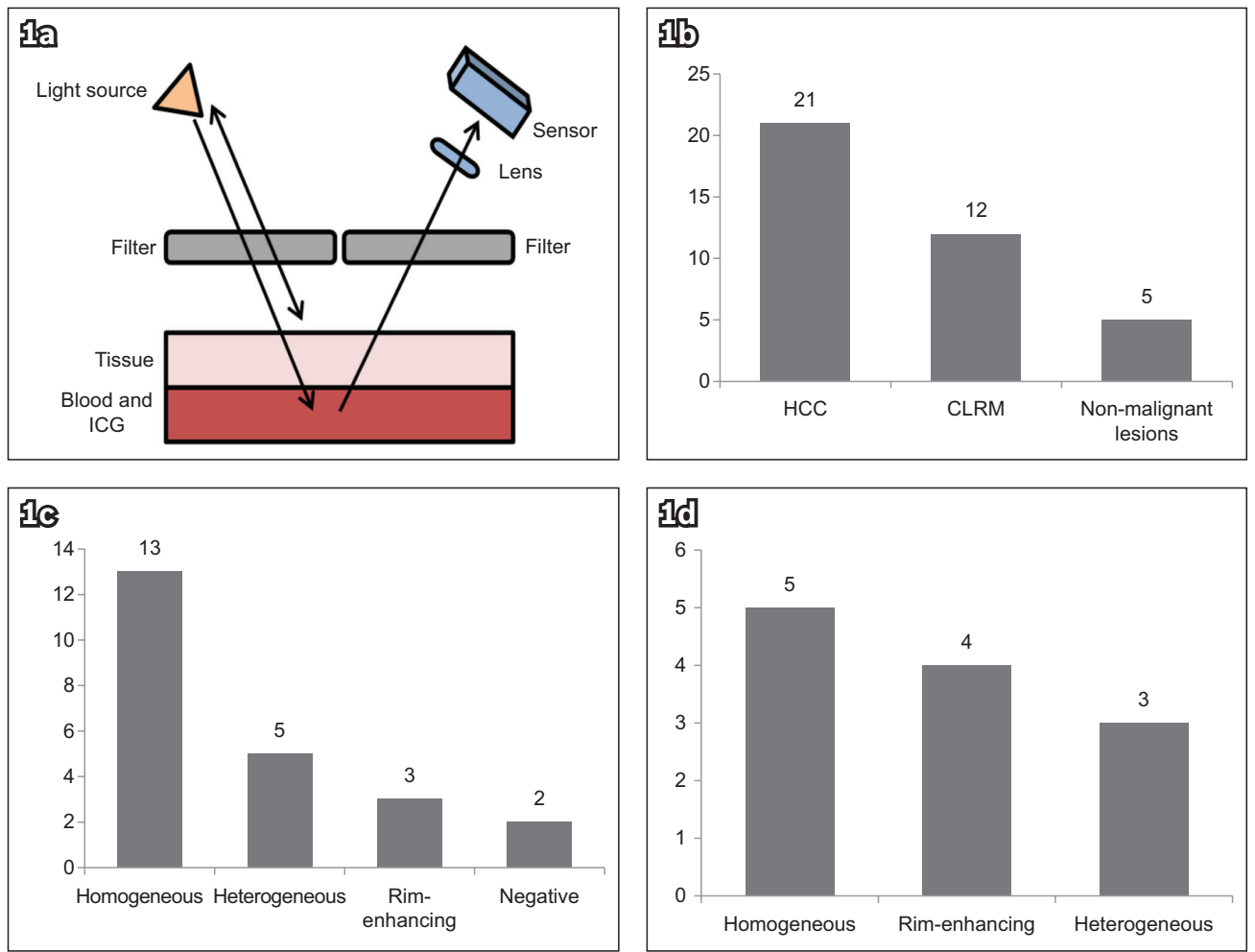

Fig. 1 (a) Diagram shows the principle of fluorescence imaging in tumour localisation: radiation from the light source is filtered by a high-pass filter to remove the fluorescent wavelengths; the blood and ICG suspension under a tissue absorbs the excitation wavelengths and emits a fluorescent band; and the emitted light is received by the sensor through a low-pass filter to remove the excitation light reflected from the source. ${ }^{(35)} \mathrm{Charts}$ show the distribution of (b) pathological types in ICG-positive lesions; (c) fluorescence patterns in hepatocellular carcinomas; and (d) fluorescence patterns in colorectal liver metastases. CLRM: colorectal liver metastases; HCC: hepatocellular carcinoma; ICG: indocyanine green

heterogeneous or rim-enhancing lesions (Fig. 3). For the lesions not detected by ICG, 6 (75.0\%) out of eight of them were in cirrhotic livers. 1 (12.5\%) out of the eight lesions was $1 \mathrm{~cm}$ deep, while the remaining $7(87.5 \%)$ were $<1 \mathrm{~cm}$ in depth. $1(50.0 \%)$ out of two lesions $\geq 1 \mathrm{~cm}$ in depth were detected by ICG, compared to 37 (84.1\%) out of 44 lesions that were $<1 \mathrm{~cm}$ in depth. The ICG-positive group had a significantly larger tumour diameter compared to the ICG-negative group (23.4 [range 4.045.0] mm vs. 8.6 [range 2.0-34.0] mm, 95\% confidence interval 1.34-28.20; $p=0.0319)$. Among the eight benign tumours, 6 $(75.0 \%)$ were negative on ICG fluorescence imaging.

Among the 38 tumours that were detected by ICG fluorescence imaging, there were 12 tumours for which ICG was administered in PRD 0-3, fewer than the 26 tumours that had ICG administered on PRD 4-7. Among the eight lesions that were ICG negative, three had ICG administered on PRD 0-3, while five had ICG administered on PRD 4-7. Nonetheless, the association between ICG detection rate and the administration regimen was not statistically significant $(p=1.000) . I C G_{R 15}$ tended to be higher in the ICG-negative group; however, this difference did not reach statistical significance (13.7 vs. $11.0,95 \% \mathrm{Cl}-4.882$ to 9.875 ; $\mathrm{p}=0.499$ ). In 15 cirrhotic patients with liver malignancies (HCC/ CLRM), those given ICG on PRD 0-3 and PRD 4-7 had detection rates of $66.7 \%$ and $91.7 \%$, respectively. The median tumour depth in the ICG-negative group tended to be higher compared to the ICG-positive group, although this did not reach statistical significance (1.5 [range 0-10.0] mm vs. 0 [range 0-6.0] mm, respectively; $p=0.308$ )

\section{DISCUSSION}

With advances in surgical techniques and medical technology, minimally invasive surgery is now a well-established approach for liver tumours. ${ }^{(27,22)}$ The fluorescence and bile excretion of ICG has been developed as a tool for real-time hepatic tumour localisation and identification, and during parenchymal transection for anatomic resection of hepatic tumours. ${ }^{(28)}$ Fusion ICG fluorescence imaging enables visualisation of pseudocolourfluorescence signals on white-light colour images and serves as a reliable localisation method, especially in laparoscopic surgery in which gross inspection and palpation may be limited. ${ }^{(29)}$ It is particularly helpful in laparoscopic surgery and in cirrhotic livers due to the challenge of distinguishing dysplastic or regenerative nodules from tumours on IOUS. This is especially difficult if the liver surface is nodular and the ultrasonography probe is unable to have a good interface on a flat surface. In such situations, if the timing of the ICG is optimised, the background liver will be dark, as the liver has had enough time for the ICG to clear and the tumour will be evident. ${ }^{(30,31)}$ Generally, for malignant lesions, the ICG tends to be retained for a much longer period than liver parenchyma, regenerative or dysplastic nodules. ${ }^{(8,20)}$ ICG tumour localisation can be very helpful for these cases, as it overcomes some of the limitations of IOUS in cirrhotic patients.

This is the first reported case series on the use of ICG fluorescence imaging for liver resection in Singapore and Southeast Asia. The present study has demonstrated its potentially useful role in the identification of subcapsular hepatic lesions, including small tumours. Our results have demonstrated that ICG 

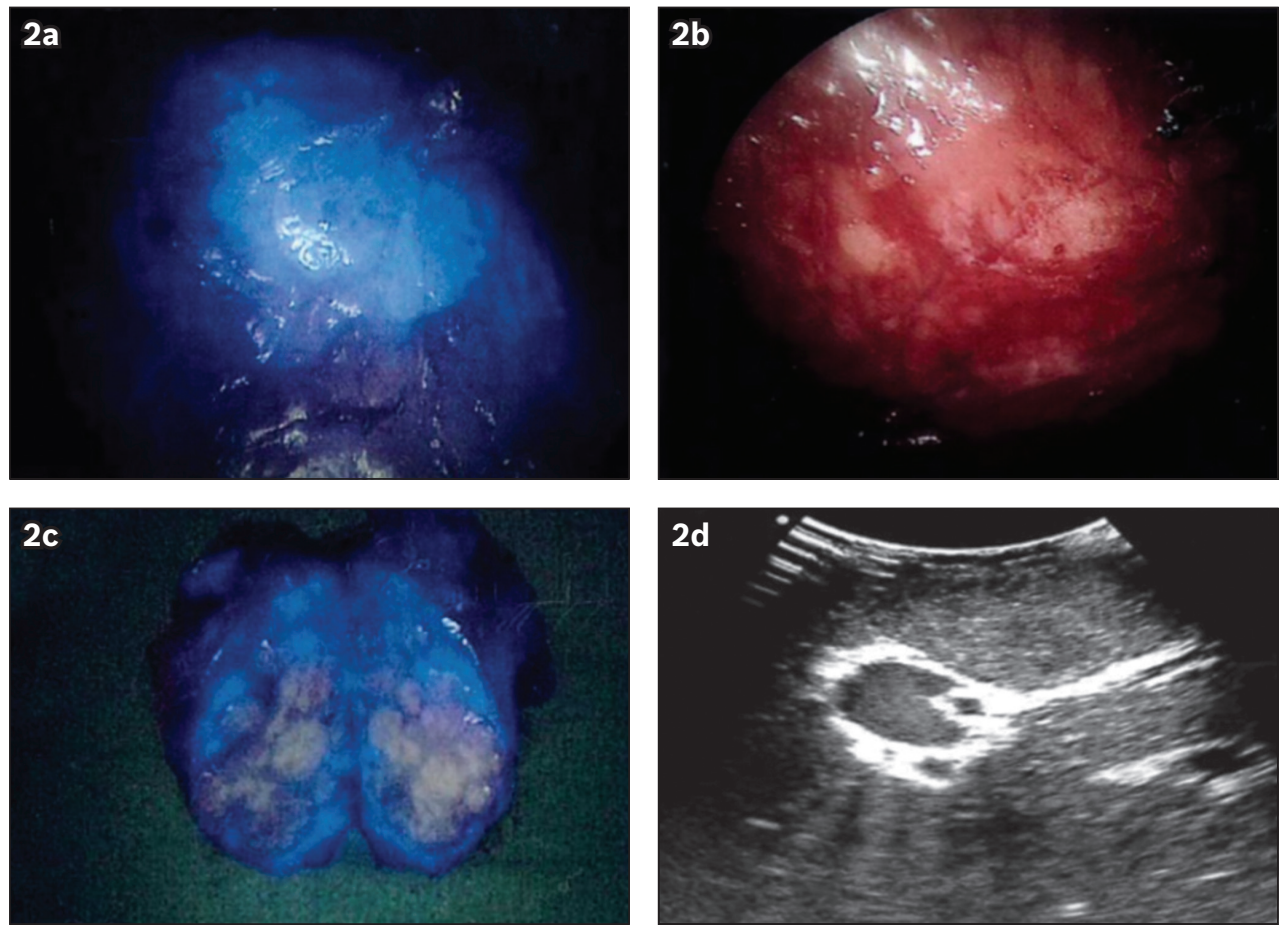

Fig. 2 A hepatocellular carcinoma (HCC) located in segment 6 during open segment 6 wedge resection. Intraoperative (a) fusion fluorescence image and (b) NIR camera photograph show (a \& b) the HCC visualised intraoperatively following a preoperative intravenous injection of indocyanine green. (c) Fluorescence image shows the resected specimen with a rim-enhancing pattern. (d) US image shows the HCC lesion intraoperatively.
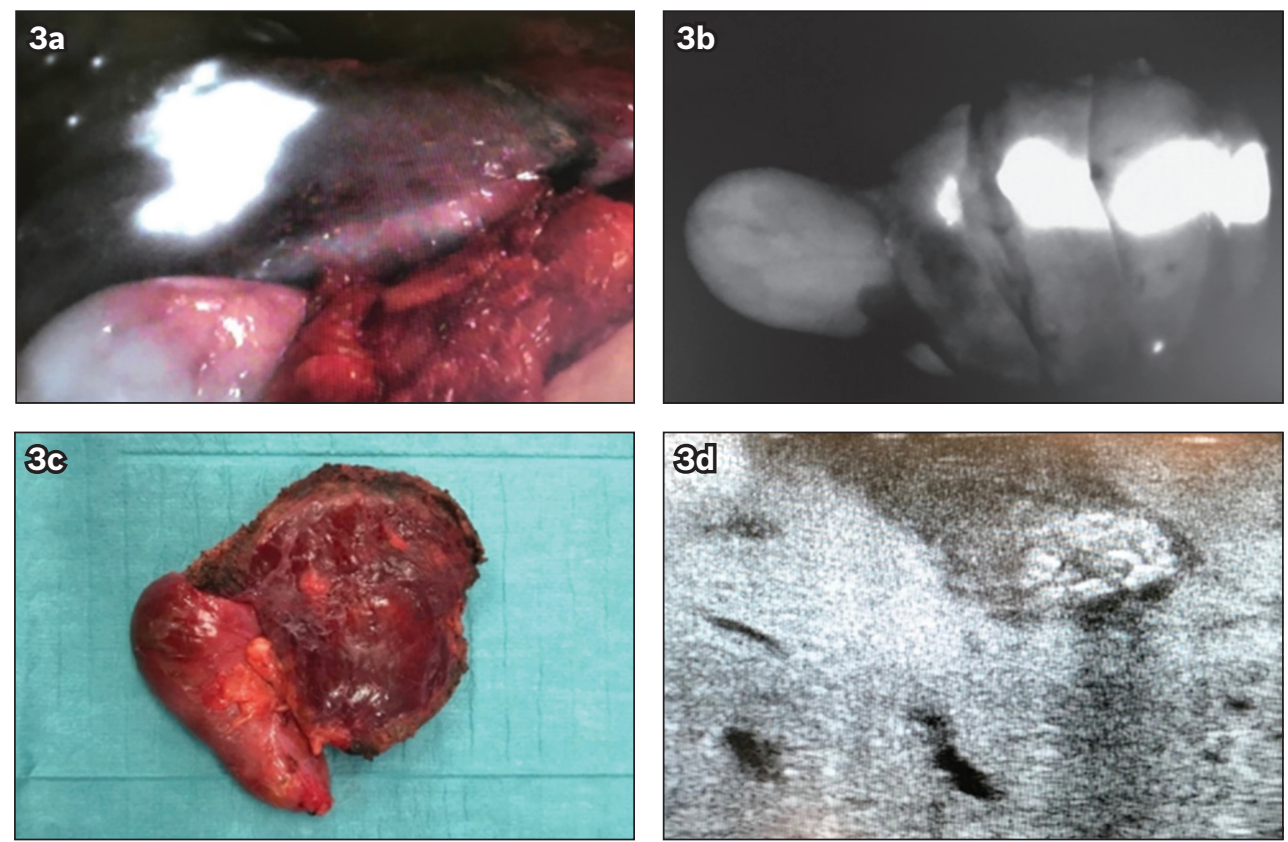

Fig. 3 A patient with colorectal liver metastasis in segment 4/5 received a preoperative intravenous injection of indocyanine green. (a) Fusion fluorescence imaging shows a homogeneous pattern clearly delineating the metastatic liver lesion. (b) Monochromatic fluorescence image shows the resected liver specimen corresponding to the metastatic liver lesion. (c) Photograph shows the resected liver specimen consisting of segment $4 / 5$ with the metastatic lesion on the liver surface. (d) US image shows the lesion intraoperatively.

fluorescence imaging is able to localise tumours clearly prior to resection in HCCs and CLRM, with characteristic fluorescent patterns for various pathological types. Notably, the majority of HCCs were detected as homogeneous fluorescing lesions, while poorly differentiated HCCs and a portion of CLRM were detected as a rim-enhancement pattern. Well- or moderately differentiated HCCs produce fluorescence as malignant tissues retain the portal uptake of ICG despite functional and architectural destruction of biliary excretion caused by tumour progression. ${ }^{(30)}$ In contrast, metastases produce a rim-enhancement fluorescent pattern as a result of impaired bile excretion in surrounding non-cancerous liver tissue that is compressed by the tumour. ${ }^{(30)}$

This imaging technique mainly highlights suspicious lesions and not benign tumours, demonstrating its specificity in identifying malignant tumours. Nonetheless, ICG fluorescence imaging has limitations in identifying deeply located tumours that 
Table III. Comparison between characteristics of the ICG-positive and ICG-negative groups.

\begin{tabular}{|c|c|c|c|c|c|c|c|}
\hline \multirow[t]{2}{*}{ Parameter } & \multicolumn{2}{|c|}{ No. (\%) } & \multirow[t]{2}{*}{ p-value } & \multirow[t]{2}{*}{ Parameter } & \multicolumn{2}{|c|}{ No. (\%) } & \multirow[t]{2}{*}{ p-value } \\
\hline & $\begin{array}{l}\text { ICG } \\
\text { positive }\end{array}$ & $\begin{array}{l}\text { ICG } \\
\text { negative }\end{array}$ & & & $\begin{array}{l}\text { ICG } \\
\text { positive }\end{array}$ & $\begin{array}{l}\text { ICG } \\
\text { negative }\end{array}$ & \\
\hline No. of lesions, patients & 38,27 & 8,5 & NA & All lesions & $1.6(0)$ & $2.6(1.5)$ & $0.308^{\S}$ \\
\hline Tumour diameter* $(\mathrm{mm})$ & $\begin{array}{l}23.4 \\
(4.0-45.0)\end{array}$ & $\begin{array}{l}8.6 \\
(2.0-34.0)\end{array}$ & 0.032 & $\begin{array}{l}\text { Pattern of } \\
\text { immunofluorescence }\end{array}$ & & & NA \\
\hline Day of ICG administration & & & & $\mathrm{HCC}$ & & $2(8.7)$ & \\
\hline $0-3$ & 12 & 3 & 1.000 & Homogeneous & $13(56.6)$ & & \\
\hline $4-7$ & 26 & 5 & & Heterogeneous & $5(21.7)$ & & \\
\hline $\mathrm{ICG}_{\mathrm{R} 15}^{+}(\%)$ & 13.7 & 11.0 & 0.499 & Rim enhancement & $3(13.0)$ & & \\
\hline Pathology & & & & CLRM & & NA & \\
\hline $\mathrm{HCC}$ & 21 & 2 & 0.003 & Homogeneous & $5(41.7)$ & & \\
\hline CLRM & 12 & 0 & & Heterogeneous & $3(25.0)$ & & \\
\hline Non-malignant & 5 & 6 & & Rim enhancement & $4(33.3)$ & & \\
\hline Percentage malignant (\%) & 86.8 & 25.0 & & Benign & & $6(54.5)$ & \\
\hline \multicolumn{4}{|c|}{ Tumour depth from capsule ${ }^{\ddagger}(\mathrm{mm})$} & Homogeneous & $1(9.1)$ & & \\
\hline $\mathrm{HCC}$ & $1.1(0)$ & $1.5(1.5)$ & $0.813^{\S}$ & Heterogeneous & $2(18.2)$ & & \\
\hline Non-malignant & $2.2(0)$ & $3.0(2.5)$ & $0.708^{\S}$ & Rim enhancement & $2(18.2)$ & & \\
\hline
\end{tabular}

Data is presented as *mean (range), tmean and $\neq$ mean (median). §p-value is calculated based on mean tumour depth between both groups. CLRM: colorectal liver metastasis; HCC: hepatocellular carcinoma; NA: not applicable

are more accurately detected by IOUS. ${ }^{(19,32)}$ As tissue penetration of the fluorescence emitted by ICG ranges from $5 \mathrm{~mm}$ to $10 \mathrm{~mm}$, it is challenging to visualise tumours that are located deeper within the liver parenchyma. In our study, the median tumour depth of ICG-negative lesions was higher compared to the ICGpositive group at $1.5(0-10.0) \mathrm{mm}$ vs. $0(0-6.0) \mathrm{mm}$, respectively, although it did not reach statistical significance, possibly limited by our small sample size. Ishizawa et al previously reported that liver tumours at a depth of $>8 \mathrm{~mm}$ from the liver surface could not be identified by ICG. ${ }^{(18)}$ In the present study, $84.1 \%(\mathrm{n}=37)$ of 44 lesions $<1 \mathrm{~cm}$ in depth were detected by ICG compared to $50.0 \%$ ( $n=1$ ) of two lesions $\geq 1 \mathrm{~cm}$ in depth. Other possible reasons for the lack of detection may include the presence of liver cirrhosis, which contributes to a bright liver background; $75.0 \%(n=6)$ of eight lesions that were not detected by ICG in our study were in cirrhotic livers. As such, it is important to understand the characteristics of the technique and to utilise it in a complementary manner with inspection and palpation or IOUS for more deeply located tumours (Table I).

Our study has also shown that it is safe to use ICG in the localisation of hepatic lesions in patients. There were no reported adverse reactions following intravenous injection of ICG in our cohort. These findings are in line with other studies that reported the utility of ICG in NIR fluorescence imaging of hepatobiliary cancer, including CLRM, HCC and cholangiocarcinoma (Table IV). ${ }^{(31,18)}$ One study showed that ICG fluorescence imaging during laparoscopic liver resection enabled highly sensitive visualisation of liver tumours and segmental boundaries where fluorescent images on a full-colour background were obtained in real time during the surgical procedure. ${ }^{(20)}$ These results demonstrated that ICG fluorescence imaging provides a simple and effective detection and navigation tool for surgeons, especially in laparoscopic liver resection. Similarly, Otsuka and Kaneko demonstrated that ICG fluorescence imaging identified subcapsular hepatic malignancies that were not diagnosed by conventional imaging modalities either preoperatively or intraoperatively. ${ }^{(21)}$ It also enabled the detection of metastatic nodules that had markedly regressed after neoadjuvant chemotherapy through visualisation of biliary excretion disorders arising in the non-cancerous hepatic parenchyma around hepatic tumours. Further support for these findings came from Zhang et al, who examined the use of fusion ICG fluorescence imaging in 41 patients undergoing laparoscopic hepatectomy and showed that among 53 malignant tumours resected, ICG imaging revealed $45(84.9 \%)$ nodules, including three nodules of CLRM that were unidentifiable by white-light colour images or IOUS. ${ }^{(33)}$ Their study also delineated the segmental boundaries on the hepatic raw surface and visceral surfaces in all 12 patients evaluated using this technique. ${ }^{(33)}$ Likewise, ICG fluorescence imaging during laparoscopic hepatectomy in 13 cases of hepatic malignancies identified $8(61.5 \%)$ lesions located close to the liver surface. ${ }^{(34)}$ This technique enabled visualisation of a colorectal liver metastasis that was not identified with preoperative imaging. ${ }^{(34,35)}$ On the whole, these findings support the feasibility of intraoperative ICG fluorescence imaging in the identification of hepatic tumours, which serves as a useful intraoperative tool.

The optimal regimen of ICG administration for liver tumour localisation is also an essential component to consider. There is currently limited literature available on the ideal timing of ICG administration for the purpose of tumour localisation. In institutions where ICG is a routine part of preoperative evaluation of liver function, these patients can benefit from NIR-ICG tumour localisation if the timing can be coordinated. In our study, the two groups that received different ICG regimens did not show 
Table IV. Summary of studies using NIR ICG fluorescence for imaging of hepatic tumours.

\begin{tabular}{|c|c|c|c|c|c|c|c|}
\hline $\begin{array}{l}\text { Study, } \\
\text { country }\end{array}$ & $\begin{array}{l}\text { No. of } \\
\text { patients }\end{array}$ & $\begin{array}{l}\text { No. of lesions } \\
\text { resected }\end{array}$ & System used & $\begin{array}{l}\text { Surgical } \\
\text { approach, ICG } \\
\text { dose }\end{array}$ & $\begin{array}{l}\text { Interval of } \\
\text { administration }\end{array}$ & Sensitivity* & Specificity $^{\dagger}$ \\
\hline $\begin{array}{l}\text { Ishizawa } \\
\text { et al,(20) Japan }\end{array}$ & $\begin{array}{l}49 ; 37 \\
\text { HCC, } \\
12 \text { CLRM }\end{array}$ & $\begin{array}{l}91 ; 63 \mathrm{HCC}, \\
28 \mathrm{CLRM}\end{array}$ & Photodynamic eye & Open, 0.5 mg/kg & $\begin{array}{l}\text { HCC 1-7 } \\
\text { (median 3) days; } \\
\text { metastases 1-14 } \\
\text { (median 3) days }\end{array}$ & $\begin{array}{l}\text { HCC } 100.0 \% \\
\text { CLRM } 100.0 \%\end{array}$ & $\begin{array}{l}\text { HCC } 93.0 \%, \\
\text { CLRM } \\
100.0 \%\end{array}$ \\
\hline $\begin{array}{l}\text { Kudo et al, }{ }^{(11)} \\
\text { Japan }\end{array}$ & 17 & $\begin{array}{l}32 ; 16 \mathrm{HCC}, \\
16 \mathrm{CLRM}\end{array}$ & Photodynamic eye & $\begin{array}{l}\text { Laparoscopic, } \\
0.5 \mathrm{mg} / \mathrm{kg}\end{array}$ & $\leq 14$ days & $\begin{array}{l}\text { HCC } 75.0 \% \\
\text { CLRM } 68.8 \%\end{array}$ & - \\
\hline $\begin{array}{l}\text { Gotoh et al, }{ }^{(17)} \\
\text { Japan }\end{array}$ & 10 & $10 \mathrm{HCC}$ & Photodynamic eye & Open, 0.5 mg/kg & 5-7 days & $100.0 \%$ & HCC $100.0 \%$ \\
\hline $\begin{array}{l}\text { Ishizawa } \\
\text { et al,(12) Japan }\end{array}$ & 1 & $1 \mathrm{HCC}$ & $\begin{array}{l}\text { Prototype imaging } \\
\text { system: Xenon light } \\
\text { source, laparoscope } \\
\text { with camera that } \\
\text { filters out light with } \\
\text { wavelengths }<810 \mathrm{~nm}\end{array}$ & $\begin{array}{l}\text { Laparoscopic, } \\
0.5 \mathrm{mg} / \mathrm{kg}\end{array}$ & 5 days & $100.0 \%$ & - \\
\hline $\begin{array}{l}\text { Kaibori } \\
\text { et al, }{ }^{(16)} \text { Japan }\end{array}$ & 116 & $116 \mathrm{HCC}$ & Clairvivo OPT & $\begin{array}{l}\text { Not mentioned, } \\
0.5 \mathrm{mg} / \mathrm{kg}\end{array}$ & $1-8 w k$ & $58.6 \%$ & - \\
\hline $\begin{array}{l}\text { Miyata } \\
\text { et al,(39) Japan }\end{array}$ & 10 & $\begin{array}{l}\text { 10; } 5 \text { HCC, } 4 \text { CLRM, } \\
1 \text { intrahepatic } \\
\text { cholangiocarcinoma }\end{array}$ & $\begin{array}{l}\text { Vevo LAZR imaging } \\
\text { system }\end{array}$ & Open, 0.5 mg/kg & Not specified & $\begin{array}{l}\text { HCC } 80.0 \% \\
\text { CLRM } 100.0 \%\end{array}$ & - \\
\hline $\begin{array}{l}\text { Present study, } \\
\text { Singapore }\end{array}$ & 32 & $\begin{array}{l}\text { 46; } 23 \text { HCC; } \\
12 \text { CLRM, } 11 \text { benign } \\
\text { lesions }\end{array}$ & $\begin{array}{l}\text { Karl Storz IMAGE } \\
\text { SPIES1 }^{\mathrm{TM}} \text { system }\end{array}$ & $\begin{array}{l}\text { Open and } \\
\text { laparoscopic, } \\
0.5 \mathrm{mg} / \mathrm{kg}\end{array}$ & $\begin{array}{l}\text { 15: } 0-3 \text { days, } \\
21: 4-7 \text { days }\end{array}$ & $\begin{array}{l}\text { HCC 9.1\%, } \\
\text { CLRM 100.0\% }\end{array}$ & $54.5 \%$ \\
\hline
\end{tabular}

*Sensitivity is defined as the correct identification of pathological subtype by ICG tumour localisation based on histologically confirmed specimens among all identified liver lesions, i.e., true positive/(true positive+false negative). †Specificity is defined as the proportion of liver lesions that were correctly identified to be benign by ICG tumour localisation, as confirmed by histological diagnosis among all identified liver lesions, i.e., true negative/(true negative+false-positive). CLRM: colorectal liver metastasis; HCC: hepatocellular carcinoma; ICG: indocyanine green

a significant difference in the detection rate of hepatic tumours. In addition, no significant difference was observed in the $I C_{R 15}$ results between the ICG-positive and ICG-negative groups. These results suggest that there may be no disadvantage in administering ICG closer to the operative date if it is more convenient, such as on PRD 0-3.

Nevertheless, in 15 cirrhotic patients with liver malignancies (HCC/CLRM) in our study, those given ICG on PRD 0-3 and PRD 4-7 had detection rates of $66.7 \%$ and $91.7 \%$, respectively. To avoid the liver background being too bright, it may be more beneficial to administer ICG after PRD 4 to PRD 7 for patients with liver cirrhosis. However, there was no statistically significant difference seen between the two groups. Similarly, another study has reported that the incidence of false positives can be reduced by not administering ICG on the day before surgery, especially in patients with decreased liver function due to cirrhosis or preoperative chemotherapy. ${ }^{(30)}$ Their results indicated that the signal intensity of non-cancerous liver parenchyma was higher in patients who had ICG administered within 24 hours before surgery, suggesting that the interval between ICG administration and surgery should be longer than two days to obtain an ideal lesion-to-liver contrast. ${ }^{(30)}$ Further evaluation in future prospective trials is required to determine the optimum regimen of ICG administration.

There are a few limitations to this study. Our study had a relatively small sample size and the majority of tumours were
HCCs. Hence, we had less comprehensive information on the use of ICG fluorescence imaging in other lesion types. Furthermore, there was a relatively small sample of each pathological type of liver tumour, which may not be representative of the wider population in terms of diversity in morphological and immunohistochemical characteristics. In addition, this was a retrospective non-randomised study, and hence potential biases and confounding factors may be present. These include differences in the baseline characteristics of patients with various pathological subtypes as well as selection bias. After establishing the feasibility and safety of ICG administration for the purpose of tumour localisation after this pilot study, we have since embarked on a prospective randomised controlled trial to examine the optimal timing of preoperative ICG administration to optimise the tumour-normal liver interface.

Nonetheless, the primary aim of this study was to evaluate the safety and feasibility of ICG tumour localisation. This technique is also valuable from the standpoint of being one of the few fluorescence imaging techniques for cancer that have reached clinical application. ${ }^{(36-39)}$ In the future, larger prospective studies evaluating the role of ICG fluorescence imaging in hepatobiliary surgery in conjunction with further technical innovation in this field would aid surgeons in performing accurate laparoscopic liver resections. Further studies are needed to determine the optimal ICG administration regime, especially in patients with chronic liver disease, which may hamper ICG elimination and 
thereby limit the contrast between the fluorescence emitted from background liver parenchyma and the lesion. The validation and optimal protocol established for this novel imaging technique can revolutionise intraoperative imaging and improve the specificity and sensitivity of liver tumour identification. This would significantly enhance surgeons' ability to locate and detect liver tumours with better certainty beyond the current limits of IOUS, especially for small tumours and cirrhotic livers.

In conclusion, ICG fluorescence imaging is a potentially useful technique that enables sensitive and specific identification of liver lesions intraoperatively. It is a safe and simple navigational tool for detecting hepatic tumours and enhancing the accuracy of liver resection. There may be no disadvantage to administering ICG closer to the operative date if it is more convenient, except for patients with liver cirrhosis. Further evaluation is planned with a large randomised controlled trial to establish the major clinical role of this imaging technique in the diagnosis and treatment of liver cancer.

\section{ACKNOWLEDGEMENTS}

We would like to acknowledge the technical expertise provided by our industry partners and their vendors for the NIR camera systems, namely Karl Storz Singapore (IMAGE SPIES1) and Humedical Pte Ltd (HyperEye system).

\section{REFERENCES}

1. Goh BKP, Lee SY, Teo JY, et al. Changing trends and outcomes associated with the adoption of minimally invasive hepatectomy: a contemporary singleinstitution experience with 400 consecutive resections. Surg Endosc 2018; 32:4658-65.

2. Koffron AJ, Auffenburg G, Kung R, Abecassis M. Evaluation of 300 minimally invasive liver resections at a single institution: less is more. Ann Surg 2007; 246:385-94.

3. Ogata F, Azuma R, Kikuchi M, Koshima I, Morimoto Y. Novel lymphography using indocyanine green dye for near-infrared fluorescence labeling. Ann Plast Surg 2007; 58:652-5.

4. Kitai T, Inomoto T, Miwa M, Shikayama T. Fluorescence navigation with indocyanine green for detecting sentinel lymph nodes in breast cancer. Breast Cancer 2005; 12:211-5.

5. Mullock BM, Shaw LJ, Fitzharris B, et al. Sources of proteins in human bile. Gut 1985; 26:500-9.

6. Hong SK, Lee KW, Kim HS, et al. Optimal bile duct division using realtime indocyanine green near-infrared fluorescence cholangiography during laparoscopic donor hepatectomy. Liver Transpl 2017; 3:847-52.

7. Kawaguchi Y, Nagai M, Nomura Y, Kokudo N, Tanaka N. Usefulness of indocyanine green-fluorescence imaging during laparoscopic hepatectomy to visualize subcapsular hard-to-identify hepatic malignancy. J Surg Oncol 2015; 112:514-6.

8. Aoki T, Yasuda D, Shimizu Y, et al. Image-guided liver mapping using fluorescence navigation system with indocyanine green for anatomical hepatic resection. World J Surg 2008; 32:1763-7.

9. Mitsuhashi N, Kimura F, Shimizu $\mathrm{H}$, et al. Usefulness of intraoperative fluorescence imaging to evaluate local anatomy in hepatobiliary surgery. J Hepatobiliary Pancreat Surg 2008; 15:508-14.

10. Miyata A, Ishizawa T, Tani K, et al. Reappraisal of a dye-staining technique for anatomic hepatectomy by the concomitant use of indocyanine green fluorescence imaging. J Am Coll Surg 2015; 221:e27-36.

11. Kudo H, Ishizawa T, Tani K, et al. Visualization of subcapsular hepatic malignancy by indocyanine-green fluorescence imaging during laparoscopic hepatectomy. Surg Endosc 2014; 28:2504-8.

12. Ishizawa T, Bandai Y, Kokudo N. Fluorescent cholangiography using indocyanine green for laparoscopic cholecystectomy: an initial experience. Arch Surg 2009; 144:381-2.
13. Ishizawa T, Zuker NB, Kokudo N, Gayet B. Positive and negative staining of hepatic segments by use of fluorescent imaging techniques during laparoscopic hepatectomy. Arch Surg 2012; 147:393-4.

14. Boni L, David G, Mangano A, et al. Clinical applications of indocyanine green (ICG) enhanced fluorescence in laparoscopic surgery. Surg Endosc 2015; 29:2046-55.

15. Shimada S, Ohtsubo S, Ogasawara K, Kusano M. Macro- and microscopic findings of ICG fluorescence in liver tumors. World J Surg Oncol 2015; 13:198.

16. Kaibori M, Matsui K, Ishizaki M, et al. Evaluation of fluorescence imaging with indocyanine green in hepatocellular carcinoma. Cancer Imaging 2016; 16:6.

17. Gotoh K, Yamada T, Ishikawa O, et al. A novel image-guided surgery of hepatocellular carcinoma by indocyanine green fluorescence imaging navigation. J Surg Oncol 2009; 100:75-9.

18. Ishizawa T, Fukushima N, Shibahara J, et al. Real-time identification of liver cancers by using indocyanine green fluorescent imaging. Cancer 2009; 115:2491-504.

19. Kawaguchi Y, Velayutham V, Fuks D, et al. Usefulness of indocyanine greenfluorescence imaging for visualization of the bile duct during laparoscopic liver resection. J Am Coll Surg 2015; 221:e113-7.

20. Ishizawa T, Saiura A, Kokudo N. Clinical application of indocyanine greenfluorescence imaging during hepatectomy. Hepatobiliary Surg Nutr 2016; 5:322-8.

21. Otsuka $\mathrm{Y}$, Kaneko H. Usefulness of ICG fluorescence imaging in laparoscopic liver resection. In: Kusano $M$, Kokudo $N$, Toi M, Kaibori $M$, eds. ICG Fluorescence Imaging and Navigation Surgery. 1st ed. Springer, 2016: 397-408.

22. Lwin TM, Hoffman RM, Bouvet M. Regarding the applications of fusionfluorescence imaging using indocyanine green in laparoscopic hepatectomy. Transl Gastroenterol Hepatol 2017; 2:70.

23. Herman $P$, Coelho FF, Perini MV, et al. Hepatocellular adenoma: an excellent indication for laparoscopic liver resection. HPB (Oxford) 2012; 14:390-5.

24. Goh BK, Lee LS, Lee SY, et al. Initial experience with robotic hepatectomy in Singapore: analysis of 48 resections in 43 consecutive patients. ANZ J Surg 2019; 89:201-5.

25. Goh BKP, Teo JY, Lee SY, et al. Critical appraisal of the impact of individual surgeon experience on the outcomes of laparoscopic liver resection in the modern era: collective experience of multiple surgeons at a single institution with 324 consecutive cases. Surg Endosc 2018; 32:1802-11.

26. Teo JY, Kam JH, Chan CY, et al. Laparoscopic liver resection for posterosuperior and anterolateral lesions-a comparison experience in an Asian centre. Hepatobiliary Surg Nutr 2015; 4:379-90.

27. Inoue Y, Arita J, Sakamoto T, et al. Anatomical liver resections guided by 3 -dimensional parenchymal staining using fusion indocyanine green fluorescence imaging. Ann Surg 2015; 262:105-11.

28. Tomassini F, Scarinci A, Elsheik $Y$, et al. Indocyanine green near-infrared fluorescence in pure laparoscopic living donor hepatectomy: a reliable road map for intra-hepatic ducts? Acta Chir Belg 2015; 115:2-7.

29. Pang TC, Lam VW. Surgical management of hepatocellular carcinoma. World J Hepatol 2015; 7:245-52.

30. Herman P, Coelho FF, Perini MV, et al. Hepatocellular adenoma: an excellent indication for laparoscopic liver resection. HPB (Oxford) 2012; 14:390-5.

31. Takahashi $\mathrm{H}$, Zaidi N, Berber E. An initial report on the intraoperative use of indocyanine green fluorescence imaging in the surgical management of liver tumors. J Surg Oncol 2016; 14:625-9.

32. Scroggie DL, Jones C. Fluorescent imaging of the biliary tract during laparoscopic cholecystectomy. Ann Surg Innov Res 2014; 8:5.

33. Zhang YM, Shi R, Hou JC, et al. Liver tumor boundaries identified intraoperatively using real-time indocyanine green fluorescence imaging. J Cancer Res Clin Oncol 2017; 143:51-8

34. Yokoyama N, Otani $\mathrm{T}$, Hashidate $\mathrm{H}$, et al. Real-time detection of hepatic micrometastases from pancreatic cancer by intraoperative fluorescence imaging: preliminary results of a prospective study. Cancer 2012; 118:2813-9.

35. Terasawa M, Ishizawa T, Mise $Y$, et al. Applications of fusion-fluorescence imaging using indocyanine green in laparoscopic hepatectomy. Surg Endosc 2017; 31:5111-8

36. Kawaguchi Y, Velayutham V, Fuks D, et al. Usefulness of indocyanine greenfluorescence imaging for visualization of the bile duct during laparoscopic liver resection. J Am Coll Surg 2015; 221:e113-7.

37. Scroggie DL, Jones C. Fluorescent imaging of the biliary tract during laparoscopic cholecystectomy. Ann Surg Innov Res 2014; 8:5.

38. Ishizawa T, Masuda K, Urano Y, et al. Mechanistic background and clinical applications of indocyanine green fluorescence imaging of hepatocellular carcinoma. Ann Surg Oncol 2014; 21:440-8.

39. Miyata A, Ishizawa T, Kamiya $M$, et al. Photoacoustic tomography of human hepatic malignancies using intraoperative indocyanine green fluorescence imaging. PLoS One 2014; 7:e112667. 